\title{
ATTRIBUTES OF DATA BREACH NOTIFICATION LAWS IN THE UNITED STATES
}

\author{
David T. Green, Governors State University dgreen@govst.edu \\ Nancy L. Martin, Southern Illinois University Carbondale nlmartin@siu.edu
}

\begin{abstract}
The United States currently does not have a federal law covering data breach notifications for most areas of commercial activity involving consumer data. In place of a common federal law, many US states have enacted laws that address data breaches to varying degrees. This paper identifies the common attributes of state laws in the US to determine common requirements that may be useful for inclusion in federal breach notification legislation.
\end{abstract}

Keywords: Data Breach, Privacy, Notification, Law, Information Security

\section{INTRODUCTION}

President Obama has called on the United States Congress to pass a national data breach law to provide "one clear national standard" because at present there is a "patchwork of state laws that dictate how companies should respond to data breaches" (Perlroth, 2014). A data breach is a compromise of security that leads to the accidental or unlawful destruction, loss, alteration, unauthorized disclosure of, or access to protected data transmitted, stored or otherwise processed" (ISO/IEC 27040).

Forty-seven states, the District of Columbia, Guam, Puerto Rico, and the Virgin Islands have passed laws requiring government and private organizations to notify individuals when a security breach has occurred involving the individual's personal information (NCSL, 2015).

Although there are many common attributes among data breach notification laws that have been enacted, there are variations in some areas.

\section{DATA BREACHES}

Hacks of consumer personal information have become a regular occurrence with security breaches impacting a wide variety of industries including banking, retail, and healthcare. There is a significant cost and time associated with individuals affected by data breaches (Gatzlaff \& McCullough, 2012), and few cases of identity theft are solved (Owens, 2014). Data breaches are "expensive and time-consuming" for organizations and individuals impacted by a breach (Gatzlaff \& McCullough, 2012).

Legislation is necessary for defining entities, organizations or individuals, who must comply; definitions of personal information; information regarding what constitutes a breach; requirements for notification; exemptions to notification; and penalties/consequences of failure to comply.

\section{METHODOLOGY}

Using a policy analysis technique, the common attributes of state security breach notification legislation were examined. The starting point was the National Conference of State Legislatures list of state security breach notification laws (NCSL, 2015). Based on an initial review of laws a set of attributes were defined that appear in 
most laws. Twenty-two laws focus on both electronic data and paper records, while twenty-four focus on electronic data records.

\section{ATTRIBUTES OF STATE DATA BREACH NOTIFICATION LAWS}

\section{Scope of the Statue - Who Must Comply?}

State legislation typically determines the categories of entities for which the law applies. Most categorize the entities as businesses, data and information brokers, and government entities, covering one or more of the categories. Fortysix laws cover business entities; thirty-five include government; and two laws specifically identify information brokers, an entity that might also be considered a business (see Table 1).

Table 1. Count of States with Privacy Laws Covering Each Entity Type

\begin{tabular}{|l|c|}
\hline Entity Type & \# Laws \\
\hline Business & 46 \\
\hline Government & 35 \\
\hline Information Brokers & 2 \\
\hline
\end{tabular}

\section{Definitions of Personal Information}

Among the legislation, 'personal information' is defined as first name, last name or usernames plus an additional piece(s) of identifiable information including social security number, state ID or drivers' license; account/credit/debit card number; or username/email plus password or security question. Some laws clarify that even if certain data has been breached the organization may not be required to notify individuals if the data breached was encrypted, unless the encryption key was also lost. Only three state laws cover encrypted personal information.

\section{What Constitutes a Breach?}

Personal information as defined by the law must either be breached or likely misused or both, triggering a notification. A breach is defined as "unauthorized access" or "unauthorized acquisition" of covered personal information maintained by a covered entity. Some definitions of a breach go into greater detail regarding exemptions for encrypted, unreadable or unusable data that may be impacted. Some laws describe how illegal use or likelihood of illegal use of the personal information. State statutes typically define a breach similar to the following: "Unauthorized acquisition of unencrypted computerized data that compromises the security, confidentiality, or integrity of personal information maintained by an individual or a commercial entity."

Notification requirements range from vague "shall be made without unreasonable delay" while giving time for the covered entity to determine the scope of the breach. Some specify 30-45 days unless law enforcement approves due to reasons related to an ongoing criminal investigation.

The method of notification typically includes one or more of the following forms of communication: written, electronic, or telephonic. Exemptions to notification or timing of notification may include:

Data was likely misused but not breached. In consultation with law enforcement, a determination may be made that the personal information misused will not result in identity theft or other harm to individuals.

Data was encrypted. If the personal information was encrypted or only included elements of the data that are unusable an exemption may be allowed.

On-Going Law Enforcement Investigation. If law enforcement determines notification would harm an ongoing investigation an exemption may be consider but often requires a written request from the law enforcement agency. 
Fifteen states do not require notification if there is a low risk of harm. The penalties of failure to notify individuals after a breach include a range from as little as no consequence at all to consequences including fines (with maximum fines ranging from $\$ 0-\$ 500,000$ ); civil action for recovering damages; and prison time. Alternatively, some laws refer to other laws covering consumer fraud. Figure 1 includes the text of all state data breach notification laws. The word cloud highlights the most frequently used words and phrases by increasing the size of the word for more frequent words.

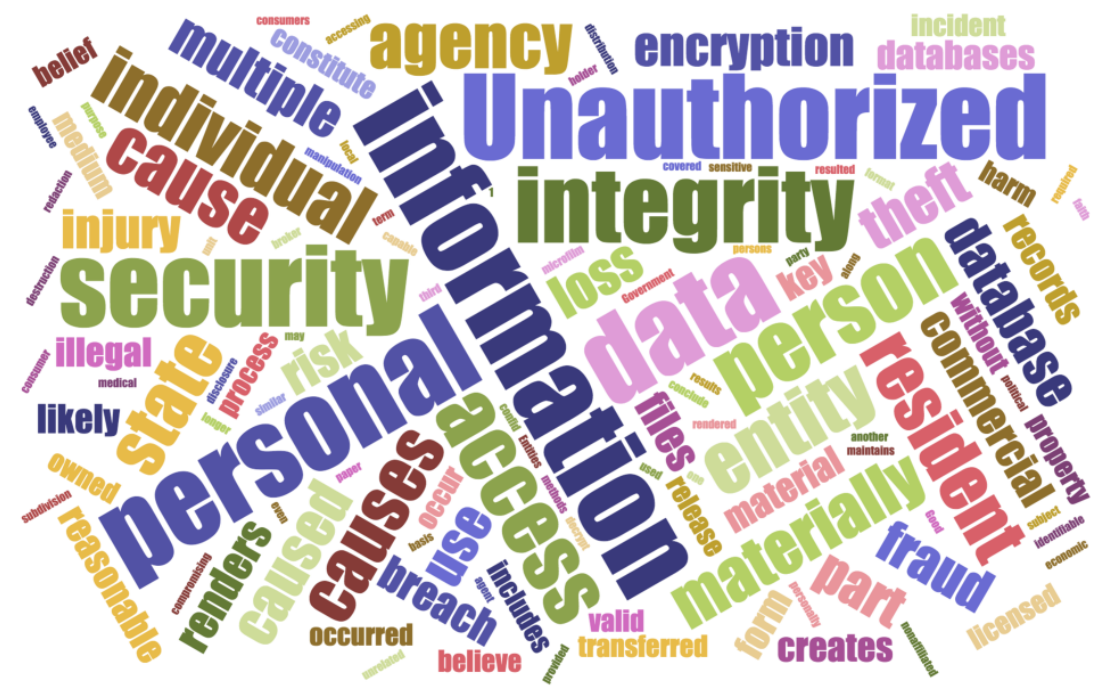

Figure 1. Word Cloud Including Data Breach Definitions from All State Privacy Laws

\section{FEDERAL}

A company operating across the nation must follow state law among 47 states and the District of Columbia. When enacted, US federal law supersedes state legislation. In the US, federal law does exist for data privacy and breach notification in a few contexts, including financial information (Gramm Leach Bliley Act; Sarbanes-Oxley Act), health information (Health Insurance Portability and Accountability Act of 1996; Health Information Technology for Economic and Clinical Health Act of 2009), and student educational records (Family Educational Rights and Privacy Act). Other consumer information used by retailers or other organizations outside healthcare and financial services is not protected by federal law in the US.

Beyond requirements of law, there may be competitive market pressures for companies to notify consumers about data breaches, but given the inconsistencies among state legislation, and the inclusion of data privacy and breach notification requirements in specific settings, including general individual data in any context is an important step to provide uniform responses and penalties within the United States. 


\section{CONCLUSION}

Based on the policy analysis of forty-seven state data breach notification laws it is clear there are differences across laws, but the general structure of each law is very similar with each including language that defines the covered entity, defines personal information, determines what constitutes a breach, requirements for notification, and penalties or consequence of failure to comply). A federal law will be next logical step because data breaches are on the rise (Williamson, 2015) and litigation occurring as a result of data breaches is increasing (Grossenbacher, 2015). A federal law would increase protections for consumers and decrease the complexity and expense for businesses attempting to comply with a patch work of laws across states. Statutes citations and summary data are included in Appendix 1 and 2.

\section{REFERENCES}

Gatzlaff, K. M., \& McCullough, K. A. (2012). Implications of Privacy Breaches for Insurers. Journal of Insurance Regulation, 31 .

Grossenbacher, K. (2015). Businesses Need a Preemptive Federal Law on Data Breach Notification. The Hill. http://thehill.com/blogs/congress-blog/judicial/248978-businesses-need-a-preemptive-federal-law-on-databreach

ISO/IEC 27040. (2015). ISO Standards Catalogue.

National Conference of State Legislatures. (2015) Security Breach Notification Laws. http://www.ncsl.org/research/telecommunications-and-information-technology/security-breach-notificationlaws.aspx

Owens, M. (2004). Policing Privacy: Michigan Law Enforcement Officers on the Challenges of Tackling Identity Theft. http://pirgim.org/reports/policingprivacy04.pdf

Perlroth, N. (December 4, 2014). Hacked vs. Hackers: Game On. New York Times Bits Blog. http://bits.blogs.nytimes.com/2014/12/02/hacked-vs-hackers-game-on/?_r=0

Federal Trade Commission. (2000). Fair Information Practices. https://www.ftc.gov/reports/privacy-online-fairinformation-practices-electronic-marketplace-federal-trade-commission

Williamson, W. (2015). Data Breaches by the Numbers. Security Week, August 31, 2015. http://www.securityweek.com/data-breaches-numbers 


\section{Appendix 1: United States Data Breach Notification Law Citations by Statute Entity}

\begin{tabular}{|c|c|c|}
\hline \multirow{2}{*}{ State } & \multicolumn{2}{|l|}{ Statute (By Entity) } \\
\hline & Business/ Information Broker & Government \\
\hline Alabama & \multicolumn{2}{|l|}{ NONE } \\
\hline Alaska & Alaska Stat. $\S 45.48 .010$ et seq. & \\
\hline Arizona & Ariz. Rev. Stat. $\S 44-7501$ & \\
\hline Arkansas & Ark. Code $\S 4-110-101$ et seq. & \\
\hline California & Cal. Civ. Code $\S \S 1798.29,1798.80$ et seq. & Cal. Civ. Code $\S \S 1798.29$, \\
\hline Colorado & Colo. Rev. Stat. § 6-1-716 & \\
\hline Connecticut & Conn. Gen Stat. § 36a-701b & \\
\hline Delaware & Del. Code tit. $6, \S 12 \mathrm{~B}-101$ et seq. & \\
\hline Florida & Fla. Stat. $\S 817.5681$ & \\
\hline Georgia & Ga. Code $\S \S 10-1-910,-911,-912$; & \\
\hline Hawaii & Haw. Rev. Stat. $\S 487 \mathrm{~N}-1$ et seq. & \\
\hline Idaho & Idaho Stat. $\S \S 28-51-104$ to -107 & \\
\hline Illinois & 815 ILCS $\S \S 530 / 1$ to $530 / 25$ & \\
\hline Indiana & Ind. Code $\S \S 4-1-11$ et seq., $24-4.9$ et seq. & \\
\hline Iowa & Iowa Code $\S \S 715$ C.1, 715C.2 & \\
\hline Kansas & Kan. Stat. $\S 50-7 \mathrm{a} 01$ et seq. & \\
\hline Kentucky & Ky. Rev. Stat. Ann. §§ $365.720-.734$ & KRS $\S \S 61.931$ to 61.934 \\
\hline Louisiana & La. Rev. Stat. $\S 51: 3071$ et seq. & \\
\hline Maine & Me. Rev. Stat. tit. $10 \S 1347$ et seq. & \\
\hline Maryland & Md. Code Com. Law $\S \S 14-3501$ et seq., & Md. State Govt. Code $\S \S 10-1301$ to -1308 \\
\hline Massachusetts & Mass. Gen. Laws $\S 93 \mathrm{H}-1$ et seq. & \\
\hline Michigan & Mich. Comp. Laws $\S \S 445.63,445.72$ & \\
\hline Minnesota & Minn. Stat. $\S \S 325 E .61,325 E .64$ & \\
\hline Mississippi & Miss. Code § 75-24-29 & \\
\hline Missouri & Mo. Rev. Stat. $\S 407.1500$ & \\
\hline Montana & Mont. Code $\S 30-14-1701$ et seq. & Mont. Code§ 2-6-504 \\
\hline
\end{tabular}




\begin{tabular}{|c|c|c|}
\hline Nebraska & $\begin{array}{l}\text { Neb. Rev. Stat. } \S \S 87-801,-802,-803,-804,-805,- \\
806,-807\end{array}$ & \\
\hline Nevada & Nev. Rev. Stat. $\S \S$ 603A.010 et seq., 242.183 & \\
\hline $\begin{array}{l}\text { New } \\
\text { Hampshire }\end{array}$ & N.H. Rev. Stat. $\S \S 359-C: 19,-C: 20,-C: 21$ & \\
\hline New Jersey & N.J. Stat. $\S 56: 8-163$ & \\
\hline New Mexico & NONE & \\
\hline New York & N.Y. Gen. Bus. Law $\S 899$-aa, & N.Y. State Tech. Law 208 \\
\hline North Carolina & N.C. Gen. Stat $\S \S 75-61,75-65$ & \\
\hline North Dakota & N.D. Cent. Code $\S 51-30-01$ et seq. & \\
\hline Ohio & Ohio Rev. Code $\S \S, 1349.19,1349.191,1349.192$ & Ohio Rev. Code $\S \S 1347.12$ \\
\hline Oklahoma & Okla. Stat. $\S \S 74-3113.1,24-161$ to -166 & \\
\hline Oregon & Oregon Rev. Stat. $\S$ 646A.600 et seq. & \\
\hline Pennsylvania & 73 Pa. Stat. $\S 2301$ et seq. & \\
\hline Rhode Island & R.I. Gen. Laws $\S 11-49.2-1$ et seq. & \\
\hline South Carolina & S.C. Code $\S 39-1-90,2013$ H.B. 3248 & \\
\hline South Dakota & NONE & \\
\hline Tennessee & Tenn. Code $\S 47-18-2107$ & \\
\hline Texas & $\begin{array}{l}\text { Tex. Bus. \& Com. Code } \S \S 521.002,521.053 \text {, Tex. } \\
\text { Ed. Code } \S 37.007(b)(5)\end{array}$ & \\
\hline Utah & Utah Code $\S \S 13-44-101$ et seq. & \\
\hline Vermont & Vt. Stat. tit. $9 \S 2430,2435$ & \\
\hline Virginia & Va. Code $\S 18.2-186.6, \S 32.1-127.1: 05$ & \\
\hline Washington & Wash. Rev. Code $\S 19.255 .010,42.56 .590$ & \\
\hline West Virginia & W.V. Code $\S \S 46 \mathrm{~A}-2 \mathrm{~A}-101$ et seq. & \\
\hline Wisconsin & Wis. Stat. $\S 134.98$ & \\
\hline Wyoming & Wyo. Stat. $\S 40-12-501$ et seq. & \\
\hline
\end{tabular}


Appendix 2: United States Data Breach Notification Law Attributes Summary

\begin{tabular}{|c|c|c|c|c|c|c|c|c|c|c|}
\hline \multirow{2}{*}{$\begin{array}{l}\text { State } \\
\text { Abbre } \\
\text { v. }\end{array}$} & \multirow[t]{2}{*}{ Entities } & \multicolumn{4}{|c|}{ Personal Information } & \multicolumn{3}{|c|}{ Notification } & \multirow[t]{2}{*}{ Max. Fine } & \multirow{2}{*}{\begin{tabular}{|c|}
$\begin{array}{c}\text { Other } \\
\text { Contents }\end{array}$ \\
$\begin{array}{c}\text { Req. for } \\
\text { protection } \\
\text { of } \\
\text { personal } \\
\text { informati } \\
\text { on when } \\
\text { discards }\end{array}$ \\
\end{tabular}} \\
\hline & & $\begin{array}{c}\text { Personal } \\
\text { Information } \\
\text { Includes } \\
\text { (First/Last } \\
\text { Name+following } \\
\text { ) }\end{array}$ & $\begin{array}{c}\text { Does Health } \\
\text { information } \\
\text { include? }\end{array}$ & Encrypted? & $\begin{array}{l}\text { Does Statute } \\
\text { Cover } \\
\text { Electronic } \\
\text { Data, Paper } \\
\text { Records, or } \\
\text { Both? }\end{array}$ & $\begin{array}{c}\text { Notice shall } \\
\text { be made } \\
\text { without } \\
\text { unreasonable } \\
\text { delay }\end{array}$ & $\begin{array}{l}\text { Notification } \\
\text { required if } \\
\text { there is a } \\
\text { Low } \\
\text { Risk of } \\
\text { Harm? }\end{array}$ & $\begin{array}{c}\text { Exemptions } \\
\text { (Maintains } \\
\text { its own } \\
\text { notification } \\
\text { procedures } \\
\text { could be } \\
\text { deemed to be } \\
\text { compliance } \\
\text { with the } \\
\text { notification } \\
\text { req.) }\end{array}$ & & \\
\hline $\mathrm{AK}$ & $\begin{array}{l}\text { Business, } \\
\text { Governme } \\
\text { nt Entities }\end{array}$ & \begin{tabular}{|c|} 
SSN, \\
State \\
ID/Drivers'License, \\
Credit/Debit Card \\
number \\
+Personal Code
\end{tabular} & No & No & Both & Yes & No & -- & 50,000 & -- \\
\hline \multicolumn{11}{|l|}{$\mathrm{AL}$} \\
\hline AR & $\begin{array}{l}\text { Business, } \\
\text { Governme } \\
\text { nt Entities }\end{array}$ & \begin{tabular}{|c|} 
SSN \\
State \\
ID/Drivers'License, \\
Credit/Debit Card \\
number \\
+Personal Code
\end{tabular} & Yes & No & $\begin{array}{c}\text { Electronic } \\
\text { Data }\end{array}$ & Yes & No & Yes & $\$ 10,000$ & Yes \\
\hline $\mathrm{AZ}$ & Business & $\begin{array}{c}\text { SSN, } \\
\text { State } \\
\text { ID/Drivers'License, } \\
\text { Credit/Debit Card } \\
\text { number } \\
\text { +Personal Code } \\
\end{array}$ & No & No & $\begin{array}{c}\text { Electronic } \\
\text { Data }\end{array}$ & Yes & No & Yes & $\$ 10,000$ & -- \\
\hline $\mathrm{CA}$ & Business & $\begin{array}{c}\text { SSN, } \\
\text { State } \\
\text { ID/Drivers'License, } \\
\text { Credit/Debit Card } \\
\text { number } \\
\text { +Personal Code }\end{array}$ & Yes & No & Both & Yes & Yes & -- & $\$ 3,000$ & -- \\
\hline $\mathrm{CO}$ & Business & $\begin{array}{c}\text { SSN } \\
\text { State } \\
\text { ID/Drivers'License, } \\
\text { Credit/Debit Card } \\
\text { number } \\
\text { +Personal Code }\end{array}$ & No & No & Both & Yes & No & Yes & -- & Yes \\
\hline $\mathrm{CT}$ & Business & $\begin{array}{c}\text { SSN, } \\
\text { State } \\
\text { ID/Drivers'License, } \\
\text { Credit/Debit Card } \\
\text { number } \\
\text { +Personal Code }\end{array}$ & No & No & $\begin{array}{c}\text { Electronic } \\
\text { Data }\end{array}$ & Yes & No & Yes & $\$ 5,000$ & -- \\
\hline $\mathrm{DE}$ & $\begin{array}{l}\text { Business, } \\
\text { Governme } \\
\text { nt Entities }\end{array}$ & \begin{tabular}{|c} 
SSN \\
State \\
ID/Drivers'License, \\
Credit/Debit Card \\
number \\
+Personal Code
\end{tabular} & No & No & $\begin{array}{c}\text { Electronic } \\
\text { Data }\end{array}$ & Yes & No & Yes & $\$ 10,000$ & -- \\
\hline FL & $\begin{array}{l}\text { Business, } \\
\text { Governme } \\
\text { nt Entities }\end{array}$ & \begin{tabular}{|c|} 
SSN \\
State \\
ID/Drivers'License, \\
Credit/Debit Card
\end{tabular} & Yes & No & Both & Yes & Yes & Yes & 500,000 & -- \\
\hline
\end{tabular}




\begin{tabular}{|c|c|c|c|c|c|c|c|c|c|c|}
\hline & & $\begin{array}{c}\text { number } \\
+ \text { Personal Code }\end{array}$ & & & & & & & & \\
\hline GA & $\begin{array}{l}\text { Business, } \\
\text { Informatio } \\
\text { n Brokers, } \\
\text { Governme } \\
\text { nt Entities }\end{array}$ & $\begin{array}{c}\text { SSN, } \\
\text { State } \\
\text { ID/Drivers'License, } \\
\text { Credit/Debit Card } \\
\text { number } \\
\text { +Personal Code }\end{array}$ & No & No & Both & Yes & Yes & -- & -- & -- \\
\hline HI & $\begin{array}{l}\text { Business, } \\
\text { Governme } \\
\text { nt Entities }\end{array}$ & $\begin{array}{c}\text { SSN, } \\
\text { State } \\
\text { ID/Drivers'License, } \\
\text { Credit/Debit Card } \\
\text { number } \\
\text { +Personal Code }\end{array}$ & No & No & Both & Yes & No & -- & 2,500 & -- \\
\hline IA & $\begin{array}{l}\text { Business, } \\
\text { Governme } \\
\text { nt Entities }\end{array}$ & $\begin{array}{c}\text { SSN, } \\
\text { State } \\
\text { ID/Drivers'License, } \\
\text { Credit/Debit Card } \\
\text { number } \\
\text { +Personal Code }\end{array}$ & No & No & Both & Yes & No & -- & $\$ 40,000$ & -- \\
\hline ID & $\begin{array}{l}\text { Business, } \\
\text { Governme } \\
\text { nt Entities }\end{array}$ & $\begin{array}{c}\text { SSN, } \\
\text { State } \\
\text { ID/Drivers'License, } \\
\text { Credit/Debit Card } \\
\text { number } \\
\text { +Personal Code }\end{array}$ & No & No & $\begin{array}{c}\text { Electronic } \\
\text { Data }\end{array}$ & Yes & No & Yes & 25,000 & -- \\
\hline IL & $\begin{array}{l}\text { Business, } \\
\text { Governme } \\
\text { nt Entities }\end{array}$ & $\begin{array}{c}\text { SSN, } \\
\text { State } \\
\text { ID/Drivers'License, } \\
\text { Credit/Debit Card } \\
\text { number } \\
\text { +Personal Code }\end{array}$ & No & No & Both & Yes & Yes & Yes & $\begin{array}{l}\$ 50,000 \\
\text { (plus an } \\
\text { additional } \\
\$ 10,000 \text { if } \\
\text { victim is } \\
65 \\
\text { years of } \\
\text { age or } \\
\text { older) }\end{array}$ & Yes \\
\hline IN & $\begin{array}{l}\text { Business, } \\
\text { Governme } \\
\text { nt Entities }\end{array}$ & $\begin{array}{c}\text { SSN, } \\
\text { State } \\
\text { ID/Drivers'License, } \\
\text { Credit/Debit Card } \\
\text { number } \\
\text { +Personal Code }\end{array}$ & No & No & Both & Yes & $\begin{array}{c}\text { No (Yes, } \\
\text { state } \\
\text { agency) }\end{array}$ & Yes & 150,000 & Yes \\
\hline $\mathrm{KS}$ & $\begin{array}{l}\text { Business, } \\
\text { Governme } \\
\text { nt Entities }\end{array}$ & $\begin{array}{c}\text { SSN, } \\
\text { State } \\
\text { ID/Drivers'License, } \\
\text { Credit/Debit Card } \\
\text { number } \\
\text { +Personal Code }\end{array}$ & No & No & Both & Yes & No & Yes & -- & -- \\
\hline KY & $\begin{array}{l}\text { Business, } \\
\text { Governme } \\
\text { nt Entities }\end{array}$ & $\begin{array}{c}\text { SSN, } \\
\text { State } \\
\text { ID/Drivers'License, } \\
\text { Credit/Debit Card } \\
\text { number } \\
\text { +Personal Code }\end{array}$ & Yes & No & Both & Yes & No & Yes & -- & Yes \\
\hline LA & $\begin{array}{l}\text { Business, } \\
\text { Governme } \\
\text { nt Entities }\end{array}$ & $\begin{array}{c}\text { SSN, } \\
\text { State } \\
\text { ID/Drivers'License, } \\
\text { Credit/Debit Card } \\
\text { number } \\
\text { +Personal Code }\end{array}$ & Yes & No & $\begin{array}{c}\text { Electronic } \\
\text { Data }\end{array}$ & Yes & No & Yes & -- & -- \\
\hline MA & $\begin{array}{l}\text { Business, } \\
\text { Governme } \\
\text { nt Entities }\end{array}$ & $\begin{array}{c}\text { SSN, } \\
\text { State } \\
\text { ID/Drivers'License, } \\
\text { Credit/Debit Card }\end{array}$ & No & No & Both & Yes & No & Yes & $\begin{array}{l}\$ 5,000, \text { or } \\
\$ 10,000 \\
\text { for } \\
\text { violating }\end{array}$ & -- \\
\hline
\end{tabular}




\begin{tabular}{|c|c|c|c|c|c|c|c|c|c|c|}
\hline & & $\begin{array}{c}\text { number } \\
+ \text { Personal Code }\end{array}$ & & & & & & & $\begin{array}{c}\text { an } \\
\text { injunction } \\
\text { entered } \\
\text { pursuant to } \\
\text { an } \\
\text { enforceme } \\
\text { nt } \\
\text { action }\end{array}$ & \\
\hline MD & $\begin{array}{l}\text { Business, } \\
\text { Governme } \\
\text { nt Entities }\end{array}$ & $\begin{array}{c} \\
\text { SSN, } \\
\text { State } \\
\text { ID/Drivers'License, } \\
\text { Credit/Debit Card } \\
\text { number } \\
\text { +Personal Code }\end{array}$ & No & No & Both & Yes & No & Yes & $\begin{array}{l}\$ 1,000 \text { for } \\
\text { first } \\
\text { violation, } \$ \\
5,000 \text { for } \\
\text { any } \\
\text { subsequent } \\
\text { violation } \\
\text { by a } \\
\text { covered } \\
\text { merchant }\end{array}$ & Yes \\
\hline $\mathrm{ME}$ & $\begin{array}{l}\text { Business, } \\
\text { Informatio } \\
\text { n Brokers, } \\
\text { Governme } \\
\text { nt Entities }\end{array}$ & $\begin{array}{c}\text { SSN, } \\
\text { State } \\
\text { ID/Drivers'License, } \\
\text { Credit/Debit Card } \\
\text { number } \\
\text { +Personal Code }\end{array}$ & No & No & $\begin{array}{c}\text { Electronic } \\
\text { Data }\end{array}$ & Yes & No & -- & 2,500 & -- \\
\hline MI & $\begin{array}{l}\text { Business, } \\
\text { Governme } \\
\text { nt Entities }\end{array}$ & $\begin{array}{c}\text { SSN, } \\
\text { State } \\
\text { ID/Drivers'License, } \\
\text { Credit/Debit Card } \\
\text { number } \\
\text { +Personal Code }\end{array}$ & No & No & $\begin{array}{c}\text { Electronic } \\
\text { Data }\end{array}$ & Yes & No & -- & 750,000 & -- \\
\hline $\mathrm{MN}$ & Business & $\begin{array}{c}\text { SSN, } \\
\text { State } \\
\text { ID/Drivers'License, } \\
\text { Credit/Debit Card } \\
\text { number } \\
\text { +Personal Code }\end{array}$ & No & No & $\begin{array}{c}\text { Electronic } \\
\text { Data }\end{array}$ & Yes & Yes & -- & $\$ 25,000$ & -- \\
\hline MO & $\begin{array}{l}\text { Business, } \\
\text { Governme } \\
\text { nt Entities }\end{array}$ & $\begin{array}{c}\text { SSN, } \\
\text { State } \\
\text { ID/Drivers'License, } \\
\text { Credit/Debit Card } \\
\text { number } \\
\text { +Personal Code }\end{array}$ & Yes & No & $\begin{array}{c}\text { Electronic } \\
\text { Data }\end{array}$ & Yes & No & Yes & -- & -- \\
\hline MS & Business & $\begin{array}{c}\text { SSN, } \\
\text { State } \\
\text { ID/Drivers'License, } \\
\text { Credit/Debit Card } \\
\text { number } \\
\text { +Personal Code }\end{array}$ & No & No & Both & Yes & No & -- & $\$ 10,000$ & -- \\
\hline MT & $\begin{array}{l}\text { Business, } \\
\text { Governme } \\
\text { nt Entities }\end{array}$ & $\begin{array}{c}\text { SSN, } \\
\text { State } \\
\text { ID/Drivers'License, } \\
\text { Credit/Debit Card } \\
\text { number } \\
\text { +Personal Code }\end{array}$ & No & -- & Both & Yes & No & -- & $\$ 10,000$ & -- \\
\hline $\mathrm{NC}$ & Business & $\begin{array}{c}\text { SSN, } \\
\text { State } \\
\text { ID/Drivers'License, } \\
\text { Credit/Debit Card } \\
\text { number } \\
\text { +Personal Code }\end{array}$ & No & No & Both & Yes & No & -- & $\$ 5,000$ & -- \\
\hline ND & Business & $\begin{array}{c}\text { SSN, } \\
\text { State } \\
\text { ID/Drivers'License, }\end{array}$ & No & No & $\begin{array}{c}\text { Electronic } \\
\text { Data }\end{array}$ & Yes & Yes & Yes & $\$ 1,000$ & -- \\
\hline
\end{tabular}




\begin{tabular}{|c|c|c|c|c|c|c|c|c|c|c|}
\hline & & $\begin{array}{c}\text { Credit/Debit Card } \\
\text { number } \\
+ \text { Personal Code }\end{array}$ & & & & & & & & \\
\hline $\mathrm{NE}$ & $\begin{array}{l}\text { Business, } \\
\text { Governme } \\
\text { nt Entities }\end{array}$ & $\begin{array}{c}\text { SSN, } \\
\text { State } \\
\text { ID/Drivers'License, } \\
\text { Credit/Debit Card } \\
\text { number } \\
\text { +Personal Code }\end{array}$ & No & -- & $\begin{array}{c}\text { Electronic } \\
\text { Data }\end{array}$ & Yes & Yes & Yes & -- & -- \\
\hline $\mathrm{NH}$ & $\begin{array}{l}\text { Business, } \\
\text { Governme } \\
\text { nt Entities }\end{array}$ & \begin{tabular}{|c} 
SSN, \\
State \\
ID/Drivers'License, \\
Credit/Debit Card \\
number \\
+Personal Code
\end{tabular} & No & No & $\begin{array}{c}\text { Electronic } \\
\text { Data }\end{array}$ & Yes & No & -- & $\begin{array}{c}\$ 10,000, \\
\text { and no less } \\
\text { than } \\
\text { double and } \\
\text { no more } \\
\text { than } \\
\text { treble } \\
\text { damages } \\
\text { in private } \\
\text { actions } \\
\text { upon } \\
\text { finding of } \\
\text { willful } \\
\text { violation }\end{array}$ & -- \\
\hline NJ & $\begin{array}{l}\text { Business, } \\
\text { Governme } \\
\text { nt Entities }\end{array}$ & $\begin{array}{c}\text { SSN, } \\
\text { State } \\
\text { ID/Drivers'License, } \\
\text { Credit/Debit Card } \\
\text { number } \\
\text { +Personal Code }\end{array}$ & No & -- & Both & Yes & No & -- & -- & Yes \\
\hline \multicolumn{11}{|l|}{ NM } \\
\hline NV & $\begin{array}{l}\text { Business, } \\
\text { Governme } \\
\text { nt Entities }\end{array}$ & $\begin{array}{c}\text { SSN, } \\
\text { State } \\
\text { ID/Drivers'License, } \\
\text { Credit/Debit Card } \\
\text { number } \\
\text { +Personal Code }\end{array}$ & No & No & Both & Yes & Yes & Yes & -- & Yes \\
\hline NY & $\begin{array}{l}\text { Business, } \\
\text { Governme } \\
\text { nt Entities }\end{array}$ & $\begin{array}{c}\text { SSN, } \\
\text { State } \\
\text { ID/Drivers'License, } \\
\text { Credit/Debit Card } \\
\text { number } \\
\text { +Personal Code }\end{array}$ & No & -- & $\begin{array}{c}\text { Electronic } \\
\text { Data }\end{array}$ & Yes & Yes & -- & $\$ 150,000$ & -- \\
\hline $\mathrm{OH}$ & $\begin{array}{l}\text { Business, } \\
\text { Governme } \\
\text { nt Entities }\end{array}$ & $\begin{array}{c}\text { SSN, } \\
\text { State } \\
\text { ID/Drivers'License, } \\
\text { Credit/Debit Card } \\
\text { number } \\
\text { +Personal Code }\end{array}$ & No & No & $\begin{array}{c}\text { Electronic } \\
\text { Data }\end{array}$ & Yes & No & -- & $\begin{array}{c}\text { penalties } \\
\text { can } \\
\text { be as high } \\
\text { as } \$ 10,000 \\
\text { per day of } \\
\text { noncompli } \\
\text { ance }\end{array}$ & -- \\
\hline $\mathrm{OK}$ & $\begin{array}{l}\text { Business, } \\
\text { Governme } \\
\text { nt Entities }\end{array}$ & $\begin{array}{c}\text { SSN, } \\
\text { State } \\
\text { ID/Drivers'License, } \\
\text { Credit/Debit Card } \\
\text { number } \\
\text { +Personal Code }\end{array}$ & No & No & $\begin{array}{c}\text { Electronic } \\
\text { Data }\end{array}$ & Yes & $\begin{array}{c}\text { No (Yes, } \\
\text { state } \\
\text { agency) }\end{array}$ & -- & 150,000 & -- \\
\hline OR & Business & $\begin{array}{c}\text { SSN, } \\
\text { State } \\
\text { ID/Drivers'License, } \\
\text { Credit/Debit Card } \\
\text { number } \\
\text { +Personal Code }\end{array}$ & No & No & Both & Yes & No & -- & $\$ 500,000$ & Yes \\
\hline
\end{tabular}




\begin{tabular}{|c|c|c|c|c|c|c|c|c|c|c|}
\hline PA & $\begin{array}{c}\text { Business, } \\
\text { Governme } \\
\text { nt Entities }\end{array}$ & $\begin{array}{c}\text { SSN, } \\
\text { State } \\
\text { ID/Drivers'License, } \\
\text { Credit/Debit Card } \\
\text { number } \\
\text { +Personal Code }\end{array}$ & No & No & $\begin{array}{c}\text { Electronic } \\
\text { Data }\end{array}$ & Yes & Yes & -- & $\$ 5,000$ & -- \\
\hline RI & $\begin{array}{l}\text { Business, } \\
\text { Governme } \\
\text { nt Entities }\end{array}$ & $\begin{array}{c}\text { SSN, } \\
\text { State } \\
\text { ID/Drivers'License, } \\
\text { Credit/Debit Card } \\
\text { number } \\
\text { +Personal Code }\end{array}$ & No & No & $\begin{array}{c}\text { Electronic } \\
\text { Data }\end{array}$ & Yes & Yes & -- & $\$ 25,000$ & -- \\
\hline $\mathrm{SC}$ & Business & $\begin{array}{c}\text { SSN, } \\
\text { State } \\
\text { ID/Drivers'License, } \\
\text { Credit/Debit Card } \\
\text { number } \\
\text { +Personal Code }\end{array}$ & No & No & $\begin{array}{c}\text { Electronic } \\
\text { Data }\end{array}$ & Yes & No & -- & $\begin{array}{l}\$ 1,000 \text { per } \\
\text { resident } \\
\text { whose } \\
\text { informatio } \\
n \text { was } \\
\text { accessible } \\
\text { if } \\
\text { violation } \\
\text { was } \\
\text { knowing } \\
\text { and } \\
\text { willful }\end{array}$ & -- \\
\hline \multicolumn{11}{|l|}{ SD } \\
\hline $\mathrm{TN}$ & $\begin{array}{l}\text { Business, } \\
\text { Governme } \\
\text { nt Entities }\end{array}$ & $\begin{array}{l}\text { SSN, } \\
\text { State } \\
\text { ID/Drivers'License, } \\
\text { Credit/Debit Card } \\
\text { number } \\
\text { +Personal Code }\end{array}$ & No & Yes & $\begin{array}{c}\text { Electronic } \\
\text { Data }\end{array}$ & Yes & Yes & -- & $\begin{array}{l}\text { The } \\
\text { greater of } \\
\$ 10,000 ; \\
\$ 5,000 \text { per } \\
\text { day of an } \\
\text { assumed } \\
\text { identity } \\
\text { theft; or } 10 \\
\text { times } \\
\text { the amount } \\
\text { obtained } \\
\text { using the } \\
\text { identity } \\
\text { theft }\end{array}$ & -- \\
\hline TX & $\begin{array}{c}\text { Business, } \\
\text { Student }\end{array}$ & $\begin{array}{c}\text { SSN, } \\
\text { State } \\
\text { ID/Drivers'License, } \\
\text { Credit/Debit Card } \\
\text { number } \\
\text { +Personal Code }\end{array}$ & No & No & Both & Yes & Yes & Yes & $\begin{array}{l}\$ 50,000, \\
\text { plus } \\
\$ 250,000 \\
\text { for failure } \\
\text { to take } \\
\text { reasonable } \\
\text { action to } \\
\text { comply } \\
\text { with notice } \\
\text { requireme } \\
\text { nts }\end{array}$ & Yes \\
\hline UT & $\begin{array}{l}\text { Business, } \\
\text { Governme } \\
\text { nt Entities }\end{array}$ & $\begin{array}{c}\text { SSN, } \\
\text { State } \\
\text { ID/Drivers'License, } \\
\text { Credit/Debit Card } \\
\text { number } \\
\text { +Personal Code }\end{array}$ & No & No & Both & Yes & No & Yes & $\$ 100,000$ & Yes \\
\hline VA & $\begin{array}{l}\text { Business, } \\
\text { Governme } \\
\text { nt Entities }\end{array}$ & $\begin{array}{c}\text { SSN, } \\
\text { State } \\
\text { ID/Drivers'License, } \\
\text { Credit/Debit Card } \\
\text { number } \\
\text { +Personal Code }\end{array}$ & Yes & Yes & $\begin{array}{c}\text { Electronic } \\
\text { Data }\end{array}$ & Yes & No & Yes & $\$ 150,000$ & -- \\
\hline
\end{tabular}


Issues in Information Systems

Volume 17, Issue I, pp. 107-118, 2016

\begin{tabular}{|c|c|c|c|c|c|c|c|c|c|c|}
\hline VT & $\begin{array}{l}\text { Business, } \\
\text { Governme } \\
\text { nt Entities }\end{array}$ & $\begin{array}{c}\text { SSN, } \\
\text { State } \\
\text { ID/Drivers'License, } \\
\text { Credit/Debit Card } \\
\text { number } \\
\text { +Personal Code }\end{array}$ & No & Yes & Both & Yes & No & -- & $\$ 10,000$ & -- \\
\hline WA & $\begin{array}{l}\text { Business, } \\
\text { Governme } \\
\text { nt Entities }\end{array}$ & $\begin{array}{c}\text { SSN, } \\
\text { State } \\
\text { ID/Drivers'License, } \\
\text { Credit/Debit Card } \\
\text { number } \\
\text { +Personal Code }\end{array}$ & No & No & $\begin{array}{c}\text { Electronic } \\
\text { Data }\end{array}$ & Yes & No & Yes & -- & -- \\
\hline WI & $\begin{array}{l}\text { Governme } \\
\text { nt Entities }\end{array}$ & $\begin{array}{c}\text { SSN, } \\
\text { State } \\
\text { ID/Drivers'License, } \\
\text { Credit/Debit Card } \\
\text { number } \\
\text { +Personal Code }\end{array}$ & No & No & Both & Yes & No & -- & $\$ 1,000$ & Yes \\
\hline WV & $\begin{array}{l}\text { Business, } \\
\text { Governme } \\
\text { nt Entities }\end{array}$ & $\begin{array}{c}\text { SSN, } \\
\text { State } \\
\text { ID/Drivers'License, } \\
\text { Credit/Debit Card } \\
\text { number } \\
\text { +Personal Code }\end{array}$ & No & No & $\begin{array}{c}\text { Electronic } \\
\text { Data }\end{array}$ & Yes & Yes & Yes & $\$ 150,000$ & -- \\
\hline WY & Business & $\begin{array}{c}\text { SSN, } \\
\text { State } \\
\text { ID/Drivers'License, } \\
\text { Credit/Debit Card } \\
\text { number } \\
\text { +Personal Code }\end{array}$ & No & No & $\begin{array}{c}\text { Electronic } \\
\text { Data }\end{array}$ & Yes & No & -- & -- & -- \\
\hline
\end{tabular}

